\title{
Sustainable Methodologies to Promote Plant Health and Post-Harvest Waste Prevention
}

\section{Jonathan Daniel Hulse*}

Department of Biology and Department of Criminology, Criminal Justice, and Sociology, Shepherd University, United States of America

*Corresponding Author: Jonathan Daniel Hulse, Department of Biology and Department of Criminology, Criminal Justice, and Sociology, Shepherd University, United States of America.
Received: June 26, 2021

Published: July 09, 2021

(C) All rights are reserved by Jonathan Daniel

Hulse.

\section{Abstract}

Sustainable agriculture is a method and philosophy of agriculture that promotes cultivation techniques that minimizes the use of synthetic pesticides as well as fertilizers in order to promote the natural processes on Earth. Promoting the use of sustainable agricultural knowledge has become increasingly popular because it utilizes the principles of ecology to encourage biological diversity, ecological diversity, and the maintenance of proper soil health. As the Earth's natural processes are better understood, agronomists, biologists, and soil scientists will become greater assets to the developing world. This manuscript is designed to disseminate knowledge about the practices of building supressive soils, as well as to foster post-harvest waste prevention, in order to make the process of getting agricultural products from the field to the table more sustainable.

Keywords: Sustainable Agriculture; Post-harvest; Waste; Suppressive-soils; Sustainability

\section{Abbreviations}

AOX: Alternative Oxidase; AMF: Arbuscular Mycorrhizal Fungi; APX: Ascorbate Peroxidase; CAT: Catalase; DHAR: Dehydroascorbate Reductase; GPX: Glutathione Peroxidase; GST: GlutathioneS-transferase; GR: Glutathione Reductase; ISR: Induced Systemic Resistance; IPM: Integrated Pest Management; MDHAR: Monodehydroascorbate Reductase; ROS: Reactive Oxygen Species; SOD: Superoxide Dismutase

\section{Introduction}

Sustainable agriculture is a multi-disciplinary approach to growing fruits, livestock, vegetables, etc. that utilizes the concepts of agronomy, ecology, microbiology, and soil science to produce food that is healthier for the planet. These sustainable agriculture techniques reduce the use of synthetic pesticides and promotes cultural practices which emulate the natural ecological processes found on Earth. Many farmers are choosing to use sustainable agricultural practices that utilized biological diversity, ecological diversity, and proper soil microbe management, to increase the longevity of their farms, as well as reduce their impact on global climate change.

Two aspects of food production that should be incorporated into sustainable agricultural practices is the building of suppressive soils, as well as the reduction in postharvest spoilage of farm products, but they are often overlooked by farmers. In the past two decades, these two marginalized facets of the sustainable agriculture field have started to gain headway, and researchers are exploring ways to integrate suppressive soils into farm management practices, as well as try and reduce waste by experimenting with innovative ways to be sustainable. 
Building suppressive soils to promote plant defense

Soil microbial communities are thought to be some of the most biologically diverse places on Earth, with more than 30,000 prokaryotic species being present in one field [6]. Soil communities are dichotomized into two distinct assemblages: suppressive soils, or conductive soils [16]. Suppressive soil types are defined by particular archaeal, bacterial and fungal communities that colonize the rhizosphere and attribute to disease resistance in plants by multiple pathways $[4,5,12]$. Conductive, or non-suppressive soils, are generally defined as soils in which diseases occurs [16]. Common suppressive soil bacteria include the following genera and families; Azospirillum spp. [Tarrand], Gluconacetobacter spp [Yamada], Bulkholderia spp [Yabuuchi], Bacillus spp [Cohn], Comamonas spp [Davis/Park], Sphingomonadaceae [Yabuuchi], Serratia spp [Bizio], Streptomyces spp [Waksman and Henrici], and Pseudomonas spp [Migula] $[4,5,13]$. Common suppressive soil fungi include; Arbuscular Mycorrhizal Fungi (AMF), non-pathogenic F. oxysporum [Synder and Hansen], Trichoderma spp [Pers], Piriformospora indica [Verma], Penicillium spp [Link], and Phoma spp [Saccardo] [4,5,12]. Classical interpretations of suppressive soils suggest that non-virulent microorganisms outcompete pathogenic microorganisms for nutrient acquisition, and outcompete niche environments, which limits the growth of pathogens [10]. Klein and colleges suggest that the host plant, as well as abiotic soil factors, and the microbial diversity in the soil, are the main criteria that characterize suppressive soils [10]. Previous studies by Klein., et al. demonstrate that organic soil amendments can induce suppressiveness in soils, which could be implemented as an integrated pest management (IPM) strategy [10]. Modern techniques used to assess suppressive soil genesis includes molecular analysis of host plant root tissues, as well as molecular identification of microbial rhizosphere communities by the means of metagenomic analysis $[10,13,16]$. Other techniques include selective media-based culturing methods, which allow for enumeration and identification of soil microbes [13]. Suppressive soils have been identified to control the following pathogens; Aphanomyces euteiches [Drechsler], Criconemella xenoplax [Luc and Raski], Fusarium oxysporum [Synder and Hansen], Gaeumannomyces graminis var. tritici [Hernandez-Restrepo], Heterodera avenae [Wollenweber], Heterodera schachtii [Schmidt], Meloidogyne spp. [Göldi], Phytophthora cinnamomic [Rands], Phytophthora infestans [Mont. De Bary], Plasmodiophora brassica e [Woronin], Pythium splendens [Hans Braun], Pythium ultimum [Trow], Rhizoctonia solani [Kühn], Ralstonia solanacearum [Smith],
Streptomyces scabies [Lambert and Loria], and Thielaviopsis basicola [Berk. and Broome] [16]. Soil suppression is dichotomized into two distinct groups; long-standing suppression, and induced suppression [16]. Long-standing suppression is characterized by unknown biological origins, which occur in soils in the absence of plants, where induced suppression is recognized to be the result of successive monoculture or the addition of inoculum to soils [4$6,16]$.

Berendsen and colleagues reviewed literature on suppressive soils, and they state that plants secrete up to $40 \%$ of their photosynthates directly into the surrounding rhizosphere, allowing for higher densities of microbial communities to form in this zone, than in uninhabited soils [6]. Plants are thought to directly influence the microbial communities that colonize their root tissues, by secreting extracellular compounds that stimulate the growth of particular bacterial and fungal species, or impede the growth of other microbial species [6].

Fusarium suppressive soils were first discovered by Atkinson in the $19^{\text {th }}$ century, and later were found to exist in other global ecosystems [16]. Non-pathogenic F. oxysporum, is thought to play a role in formation of suppressive soils by competing for nutrients with pathogenic F. oxysporum [12]. Gupta states that the bacterial clade, actinomycetes, have a great potential in reducing disease caused by soil-borne pathogens, but the extent of this protection is unknown [9]. Bakker., et al. 2013 suggests that the production of redox-active phenazines play a role in the synthesis of Fusarium suppressive soils [4,5]. In Weller's 2002 review, it is stated that Fusarium suppressive soils have been documented to extend from continuous mono cropping of resistant cultivars, which contradicts the notion that suppressive soils arise from continuous mono cropping of susceptible cultivars [16]. Weller and colleagues also suggest that the Fusarium soil suppression reduced disease incidence derived from the induced systemic resistance (ISR) pathway, but the mechanisms might be different than rhizobacteria induced systemic resistance [16].

Jasmonic/salicylic acid application for postharvest spoilage prevention

Postharvest spoilage is a major economic setback that occurs after crop harvest, during transportation, storage, and processing, which is often overlooked by consumers [7]. Many saprophytic bacteria and fungi are responsible for post-harvest losses, includ- 
ing the following fungi: Alternaria alternata [Fr. Keissl], Colletotrichum gloeosporioides [Stoneman/Spauld/Schrenk], Rhizopus stolonifera [Vuillemin], Monilinia fructicola [Winter/Honey], Botrytis cinerea [Pers.], Penicillium spp. [Link], Aspergillus spp [Micheli]. [1$3,7,8,11,14,15,17]$. Since the environmental movement, the populous has had concerns about the latent health and environmental effects of synthetic pesticide residues on food crops [1,2,7]. Scientists have been dedicated to developing post-harvest treatment applications that do not rely on synthetic chemicals but use naturally occurring defensive compounds for post-harvest protection $[1,2,7]$. Post-harvest application of salicylic acid, as well as jasmonic acid derivatives, has been shown effective at controlling saprophytic fungi growth on harvest fruits [1-3,7,8,11,14,15,17]. It is suggested that exogenous treatments of salicylic acid could be used to increase fungal resistance for cherry, mango, pear, plum, pineapple, peach, strawberry, and tomato [1,2,7]. Aghdam., et al. have written many manuscripts on the application of postharvest salicylates and jasmonates on the biological, physiological, and molecular mechanisms that promote chilling injury resistance in various flowers, fruits, and vegetables [1,2]. Aghdam's manuscripts emphasize the follow topics: chilling injury and its impact on the cell membrane, chilling injury and oxidative stress, and mechanism employed by salicylates and jasmonates for alleviating of chilling injury in fruits, vegetables, and cut flowers $[1,2]$. Aghdam states that plants have two main ways of dealing with oxidative stress; activation of genes that code for proteins that are involved in the minimizing of reactive oxygen species, such as the alternative oxidase (AOX) pathway, or by inducing the gene expression for reactive oxygen species (ROS) scavenging antioxidants such superoxide dismutase (SOD), catalase (CAT), ascorbate peroxidase (APX), glutathione peroxidase (GPX), glutathione-S-transferase (GST), monodehydroascorbate reductase (MDHAR), dehydroascorbate reductase (DHAR) and glutathione reductase (GR) [1,2].

Post-harvest application of salicylic acid has been shown to increase anti-oxidant activity of Zizyphus jujuba [Mill.], and may increase the health benefit of other fruits and vegetables [7]. Zizyphus jujuba fruit was dipped in $2 \mathrm{mmol}$ salicylic acid and stored at $0 \mathrm{C}$ for 2 months [7]. After numerous sampling events, postharvest dipping in salicylic acid proved to provide increased levels of anti-oxidants for a two-month duration, compared to the control treatments [7]. At the end of the storage period, the treated fruit showed a $16.8 \%$ increase in anti-oxidant concentration compared to the control treatments [7]. Similar experiments with pesticide free cucumbers would provide valuable information about the level of anti-oxidants in cucumbers treated with postharvest applications of salicylic acid. Post-harvest application of salicylic acid is an important step in a post-harvest integrated pest management strategy for members of the Cucurbitaceae.

\section{Conclusion}

This manuscript is intended to spread knowledge about the practice of building suppressive soils, as well as to foster post- harvest waste reduction with the use of scientifically researched sustainable agricultural techniques. The process of getting agricultural products from the field, to the table, by using these sustainable practices, will allow for a healthier agricultural ecosystem.

\section{Acknowledgements}

I want to thank Clemson University for providing internal funding during my time as a graduate student, which allowed for the writing of the majority of this manuscript. I also want to thank Shepherd University for supporting me while I finished writing this manuscript. Many thanks to Dr. Caye Drapcho and Dr. Geoff Zehnder from Clemson University for advising me in the writing of this manuscript.

\section{Conflict of Interest}

There are no conflicts of interest in the writing of this manuscript.

\section{Bibliography}

1. Aghdam Morteza Soleimani., et al. "Enhancement of antioxidant capacity of cornelian cherry (Cornus mas) fruit by postharvest calcium treatment". Scientia Horticulturae 161 (2013): 160-164.

2. Aghdam Morteza Soleimani and Samad Bodbodak. "Physiological and biochemical mechanisms regulating chilling tolerance in fruits and vegetables under postharvest salicylates and jasmonates treatments". Scientia Horticulturae 156 (2013): 7385.

3. Babalar Mesbah., et al. "Effect of pre-and postharvest salicylic acid treatment on ethylene production, fungal decay and overall quality of Selva strawberry fruit". Food Chemistry 105.2 (2007): 449-453. 
4. Bakker Peter AHM., et al. "Induced systemic resistance and the rhizosphere microbiome". The Plant Pathology Journal 29.2 (2013): 136.

5. Bakker Peter AHM., et al. "The rhizosphere revisited: root microbiomics". Frontiers in Plant Science 4 (2013): 165.

6. Berendsen Roeland L., et al. "The rhizosphere microbiome and plant health". Trends in Plant Science 17.8 (2012): 478-486.

7. Cao Jiankang., et al. "Effects of postharvest salicylic acid dipping on Alternaria rot and disease resistance of jujube fruit during storage". Journal of the Science of Food and Agriculture 93.13 (2013): 3252-3258.

8. Dokhanieh Afsaneh Yousefpour., et al. "Postharvest salicylic acid treatment enhances antioxidant potential of cornelian cherry fruit". Scientia Horticulturae 154 (2013): 31-36.

9. Gupta Vadakattu VSR. "Beneficial microorganisms for sustainable agriculture”. Microbiology Australia 33.3 (2012): 113-115.

10. Klein Eyal., et al. "Soil suppressiveness to Fusarium disease: shifts in root microbiome associated with reduction of pathogen root colonization". Phytopathology 103.1 (2013): 23-33.

11. Lu Xinhua., et al. "Pre-and post-harvest salicylic acid treatments alleviate internal browning and maintain quality of winter pineapple fruit". Scientia Horticulturae 130.1 (2011): 97-101.

12. Mandeel Qaher and Ralph Baker. "Mechanisms involved in biological control of Fusarium wilt of cucumber with strains of nonpathogenic Fusarium oxysporum". Phytopathology 81.4 (1991): 462-469.

13. Meng QX., et al. "Characterizing a novel strain of B acillus amyloliquefaciens BAC 03 for potential biological control application". Journal of Applied Microbiology 113.5 (2012): 11651175.

14. Panahirad Sima., et al. "Postharvest Control of R hizopus stolonifer in Peach (P runus persica L. B atsch) Fruits Using Salicylic Acid”. Journal of Food Safety 32.4 (2012): 502-507.

15. Shafiee M., et al. "Addition of salicylic acid to nutrient solution combined with postharvest treatments (hot water, salicylic acid, and calcium dipping) improved postharvest fruit quality of strawberry". Scientia Horticulturae 124.1 (2010): 40-45.

16. Weller David M., et al. "Microbial populations responsible for specific soil suppressiveness to plant pathogens". Annual Review of Phytopathology 40.1 (2002): 309-348.

17. Zhang Dianpeng., et al. "Efficacy of the antagonist Aureobasidium pullulans PL5 against postharvest pathogens of peach, apple and plum and its modes of action". Biological Control 54.3 (2010): 172-180.

Volume 5 Issue 8 August 2021

(C) All rights are reserved by Jonathan Daniel Hulse. 